\title{
Change Vulnerability Forecasting for Southeast Asia using Deep Learning Algorithm
}

\author{
Amelia Ritahani Ismail ${ }^{\text {a, }}$ 1, *, Nur 'Atikah Binti Mohd Ali ${ }^{\text {a }}$, Junaida Sulaiman ${ }^{\text {b, } 2}$ \\ ${ }^{a}$ Department of Computer Science, Kulliyyah of Information and Communication Technology, \\ International Islamic University Malaysia, P.O. Box 10, 50728 Kuala Lumpur, Malaysia \\ ${ }^{b}$ Soft Computing and Intelligent System (SPINT), Faculty of Computer Systems \& Software Engineering, \\ Universiti Malaysia Pahang, Lebuhraya Tun Razak, 26300, Kuantan, Pahang, Malaysia \\ ${ }^{1}$ amelia@iium.edu.my*; ${ }^{2}$ junaida@ump.edu.my \\ * corresponding author
}

Article history:

Received 19 April 2018

Revised 6 August 2018

Accepted 14 August 2018

Published online 31 August 2018

Keywords:

Deep Learning

Forecasting

Climate change

Climate change is expected to change people's livelihood in significant ways. Several vulnerability factors and readiness factors used for measuring the prediction index of that particular country on how vulnerable of a country towards global change. Primary data was collected from University of Notre Dame Global Adaptation Index (NDGAIN). The data has been trained for the forecasting purpose with support from the validated statistical analysis. The summary of the predicted index is visualized using machine learning tools. The results developed the correlation between vulnerability and readiness factors and shows the stability of the country towards climate change. The framework is applied to synthesize findings from Prediction index studies in South East Asia in dealing with vulnerability to climate change.
\end{abstract}

This is an open access article under the CC BY-SA license (https://creativecommons.org/licenses/by-sa/4.0/).

\section{Introduction}

South East Asia geographically located in the place that highly affected by the climate change. Particularly draughts, floods and tropical cyclones. Most of the area located at the ocean coast is highly prone to flash floods which are influenced by the monsoons [1][2]. The livelihood of the poor in these areas will be most threatened by these problems due to their limited adaptive capacities [2]. On the other hand, some countries already have the adaptive action by utilizing the capital investment for both public and private sectors through the cooperation between the government and the communities [3].

Most countries in Southeast Asia depend so much on climate to support their life. The change in climate means a change in their life as well whether it is for good or bad. For example, changes in rainfall occurrences and variability affect economics and infrastructure in countries such as Indonesia and Malaysia [1][2]. There are a lot of studies with different approaches have been conducted to see how much the climate change vulnerability affect their life [3]. The results show different outcomes in European countries based on the readiness factors. The level of readiness toward climate change in the developed country is good to moderate [3]. On the other hand, the developing country has under average level of readiness due to lack of access to climate information [2].

Community and its supporting agency are not really aware about the climate change towards their country in the future. Therefore, they do not well prepared for any circumstances including foods, water, habitation, income per capita, infrastructures, health and governance. When the climate change occurs, the most affected sector is agriculture because it totally depends on the climate. The worst case is that other sectors are depending on it. Therefore, they cannot work properly and the condition for every factor of that country will be in trouble.

This study uses machine learning approach to predict of climate change vulnerability in South East Asia. Recently, researchers are interested to use deep learning method in malware detection [4], green energy efficiency [5], marine ecosystems classification [6] and weather forecasting [7]. Deep learning 
allows computational models that are composed of multiple processing layers to learn representations of data with multiple levels of abstraction [8]. This method improved the data learning process which used back propagation algorithm with multiple hidden layers and iterations to discover how it changed the internal parameters by computing the previous layers. Deep learning is also able to classify unlabeled data which are difficult to handle by supervised machine learning methods such as support vector machine, decision trees and naïve Bayes [4]. By using progressive learning algorithm [8], data mining task is more effective in recognizing hidden patterns in large data.

In this paper, the prediction index measures the correlation between the vulnerability factors and the readiness factors. The elements of the vulnerability are capacity, ecosystem, exposure, food, habitat, health, infrastructure, sensitivity, and water. While for readiness are economic, governance and social.

\section{Methods}

The data used in this study was collected from University of Notre Dame Global Adaptation Index (ND-GAIN) [9]. The data is about the readiness and vulnerability index for Southeast Asia within 5 years. The data will be used to predict both indexes for another three years $(2015-2017)$. Furthermore, the data generated will be used as a reference for countries to increase their country's resilience toward climate change. The description of the elements used for the experiments based on the dataset is shown in Table 1.

To survive the climate change, the government and its supporting agencies need to improve these factors by increasing the readiness level of their country. It can be achieved through well communication and cooperation between the community, supporting agencies and the government itself. This study also provides data that can be used as a part of references for the decision making in implementing some actions for the development of that country. There are six procedures in acquiring the predictive index.

The first step is Data Acquisition. Data collected from the University of Notre Dame Global Adaptation Index (ND-GAIN). They summarized a country's vulnerability to climate change and other global challenges in combination with its readiness to improve resilience.

The second step is Data Training. The collected data from 1995 until 2014 is transformed using windowing time series forecasting with the window size of 5 . Windowing is a mathematical function with zero-valued outside its chosen interval [10]. This operator transforms a given example set containing series data into a new example set which contains single valued examples4. Windows with a specified window and step size are moved across the series and the attribute value is lying horizon values after the window end is used as label, which should be predicted. Then, the windowed data is trained using deep learning model to get the desired output. With the multiple numbers of input nodes,

Table 1. Description of the elements

\begin{tabular}{|c|c|}
\hline Element & Description \\
\hline Capacity & $\begin{array}{l}\text { The society capabilities together with the support from both public and private sectors to mitigate the } \\
\text { potential distraction and to counter the negative outcome of climate affair. }\end{array}$ \\
\hline Ecosystem & $\begin{array}{l}\text { The capability of the society and its supporting sectors to maintain the relationship between organism } \\
\text { and environment. }\end{array}$ \\
\hline Exposure & The awareness of the society and its supporting sectors toward the climate condition. \\
\hline Food & The capability of the society and its supporting sectors to maintain the food sustainability. \\
\hline Habitat & The degree of habitat resilience to climate change. \\
\hline Health & The readiness of the society and its supporting sectors to the climate change in term of health. \\
\hline Infrastructure & The readiness of the infrastructure to respond the threats of climate change. \\
\hline Sensitivity & The dependency level of society towards the affected areas caused by climate change. \\
\hline Water & The capability of the society and its supporting sectors to maintain the water supply. \\
\hline Economic & The investment from the supporting sectors to facilitate the mobilizing capitals \\
\hline Governance & $\begin{array}{l}\text { The government level of assurance to secure the invested capitals to grow with help of public services } \\
\text { without any disturbance. }\end{array}$ \\
\hline Social & $\begin{array}{l}\text { Social condition for the utilization of the investment by the government to help the society with } \\
\text { efficient and equitable usage }\end{array}$ \\
\hline
\end{tabular}


15 input nodes for readiness model, 45 input nodes for the vulnerability model and 50 hidden nodes with 25 epochs, the output can be obtained. The output layer resulted in the value for forecasted data and it went through some performance model and had provided its accuracy.

The next step is Data Testing. The trained data be tested for the next three years $(2015-2017)$ by using RapidMiner. It is a software application for visualizing the programming environment to build complete predictive analytic workflows. It is a tool for predefined data preparation and machine learning algorithm to support the data science needs. It can be used for various tasks in data analysis process such as data preparation, data training, data testing, data validation and data visualization.

The fourth step is Index Predicting. The predicted index is gained from deep learning prediction method. First, the training data is processed using a windowing series with the size of 5. Windowing is suitable for time series forecasting in RapidMiner. It allows the user to take any time series data and transform it into a cross-sectional format. After that, the windowing results are validated using the deep learning algorithm with 29 epochs. Deep learning is used for multiple running processes. It is the improvement of the backpropagation algorithm. After running the algorithm, the results performance is calculated to gain the percentage value of the validation. Finally, the model for training data is applied to the testing data to get the predicted value for both vulnerable and readiness level of each country. After that, the predicted score for each country is calculated by using Equation 1.

Score $=($ Readyness - Vulnerability +1$) * 50$

Index Validation is the fifth step. Validating the predicted data for 2015 until 2017. Checking whether it is correct or not. If the predicted data is the same with the real data, then it is true.

In the last step, Index Visualization, the information is described through visual rendering. The obtained result will be visualized using software called Tableau to make it more understandable and presentable. Tableau is a software application that is widely used in business intelligence for an organization to see and understand their data. It connects and visualizes the data that has been analyzed in a meaningful way by drag and drop the attributes which can be shared and published publicly on the web. Fig. 1 and Fig. 2 show part of visualizations of the predicted index.

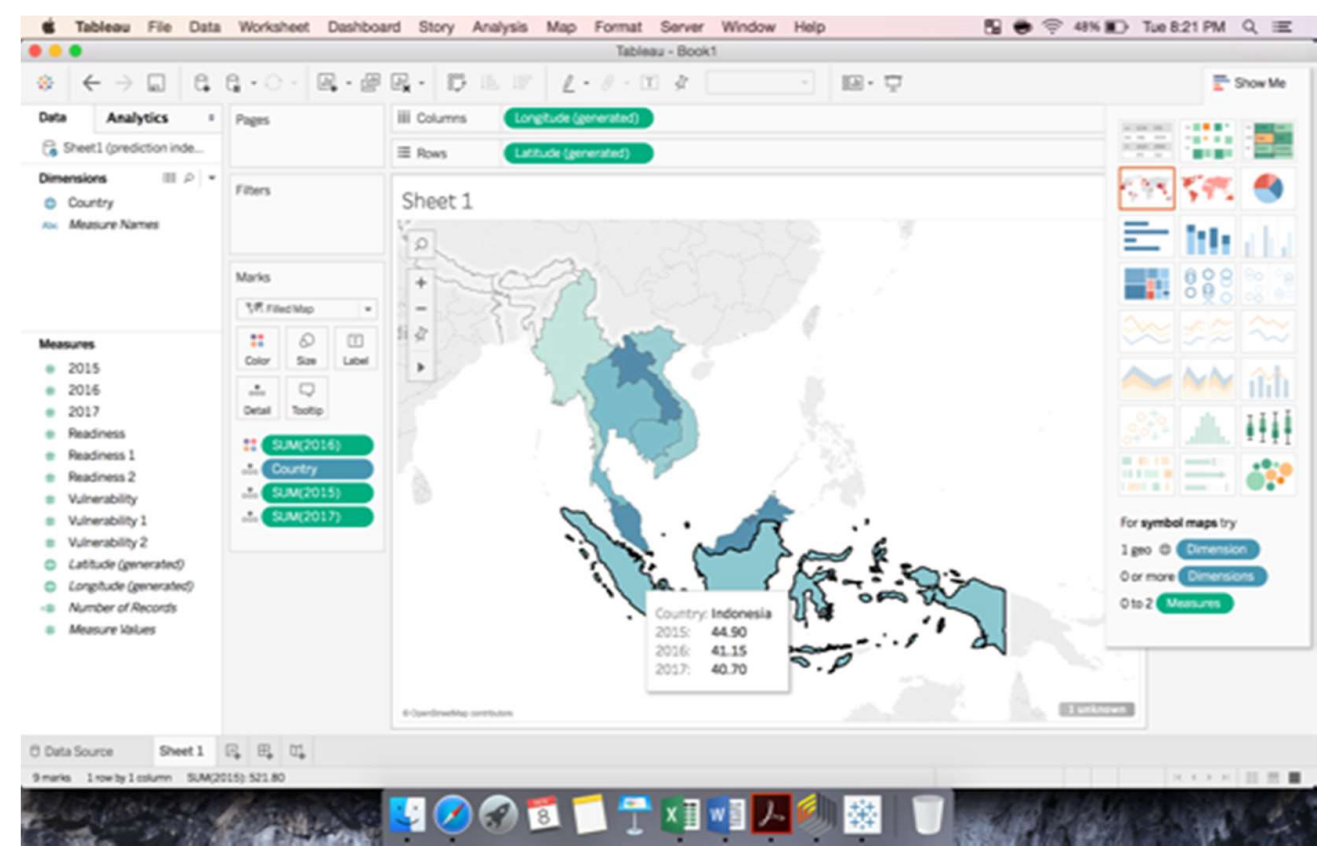

Fig. 1. Visualizing the forecasted adaptation index using Tableau 


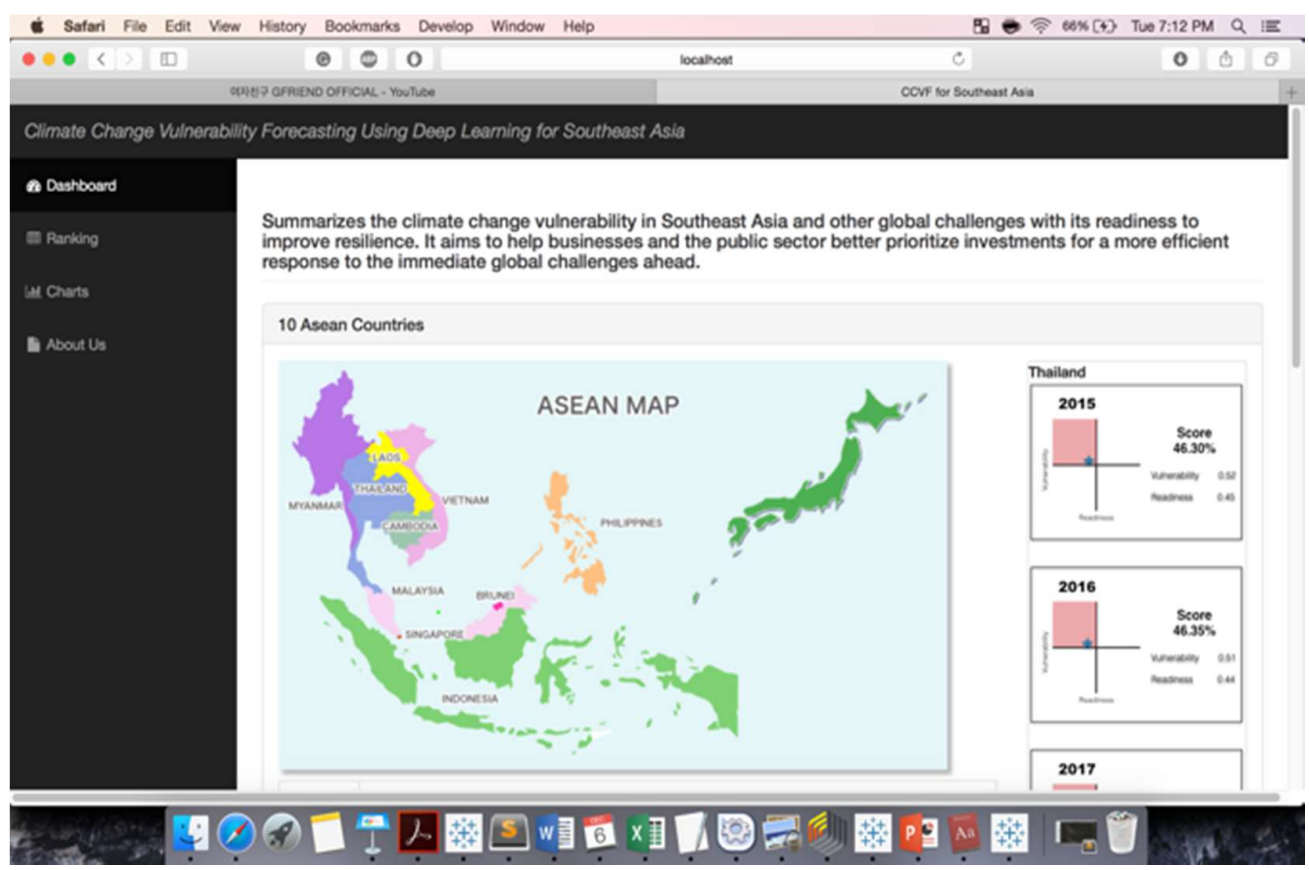

Fig. 2. Deployment of the results on the web

Table 2. Average prediction value

\begin{tabular}{lr}
\hline Experiment parameters & Predicted value \\
\hline Readiness & 0.467 \\
Vulnerability & 0.46 \\
ND-gain & 51.767 \\
Readiness-prediction & 0.476 \\
Vulnerability-prediction & 0.493 \\
Prediction Index & 49.175 \\
P Value Readiness & 0.41 \\
P Value Vulnerability & 0.013 \\
Accuracy Readiness & 0.68 \\
Accuracy Vulnerability & 0.564 \\
\hline
\end{tabular}

\section{Experimental Result}

After doing the experiment by using the deep learning approach, the predicted adaptation index for each Southeast Asia country is obtained. It is found that each country has different value for its predicted vulnerability index and readiness index as well as the performance accuracy. After that, the predicted index vulnerability and readiness will go through the statistical analysis to validate either to accept or to reject the hypothesis.

When validating the country's vulnerability and readiness level based on the five years' data to prove the deep learning study as in Table 1 , it is found $60 \%$ of the countries rejected the hypothesis, those are Indonesia, Laos, Malaysia, Myanmar, Philippines, and Singapore. The obtained values from the Wilcoxon Rank Sum test are 0.3095, 0.1493, 0.3095, 0.1337, 0.1587 and 0.09524 respectively. It means that the predicted value has the smallest different compared to the real data and the results are promising which leads to high potential to be accepted. While the other $40 \%$ are from Brunei, Cambodia, Thailand, and Vietnam accepted the hypothesis because their predicted values are significantly different compared to the real data. 


\section{Conclusions}

The study conducted has proved that there is a slight different in the result using deep learning analysis with the original given data. Based on the findings it is the truth that the predicted vulnerability and readiness values obtained using deep learning do differ and affect the adaptation index of the countries. Therefore, the government and any private sectors should take actions in order to prepare for any critical aspects in terms of life, economic and others that can affect the population of the country.

\section{References}

[1] Hardwinarto, Sigit, and Marlon Aipassa. "Rainfall Monthly Prediction Based on Artificial Neural Network: A Case Study in Tenggarong Station, East Kalimantan-Indonesia." Procedia Computer Science 59 (2015): 142-151.

[2] Sulaiman, Junaida Binti, Herdianti Darwis, and Hideo Hirose. "Monthly Maximum Accumulated Precipitation Forecasting Using Local Precipitation Data and Global Climate Modes." Journal of Advanced Computational Intelligence and Intelligent Informatics 18.6 (2014): 999-1006.

[3] Ezra, C. Alyosha. "Climate Change Vulnerability Assessment in the Agriculture Sector: Typhoon Santi Experience." Procedia-Social and Behavioral Sciences 216 (2016): 440-451.

[4] Yuxin, Ding, and Zhu Siyi. "Malware detection based on deep learning algorithm." Neural Computing and Applications (2017): 1-12.

[5] Wang, Huai-zhi, Gang-qiang Li, Gui-bing Wang, Jian-chun Peng, Hui Jiang, and Yi-tao Liu. "Deep learning based ensemble approach for probabilistic wind power forecasting." Applied Energy 188 (2017): 56-70.

[6] A. Grover, A. Kapoor, and E. Horvitz, "A Deep Hybrid Model for Weather Forecasting," Proceedings of the $21^{\text {th }}$ ACM SIGKDD International Conference on Knowledge Discovery and Data Mining - KDD '15, 2015

[7] Y. Lecun, Y. Bengio, and G. Hinton, "Deep learning," Nature, vol. 521, no. 7553, pp. 436-444, 2015.

[8] A. G. Salman, B. Kanigoro, and Y. Heryadi, "Weather forecasting using deep learning techniques," 2015 International Conference on Advanced Computer Science and Information Systems (ICACSIS), 2015.

[9] “ND-GAIN Index,” ND-GAIN Index. [Online]. Available: http://index.gain.org/. [Accessed: 06-Oct-2016].

[10] B. Deshpande, "Time Series Forecasting: from windowing to predicting in RapidMiner," http://www.simafore.com/blog/bid/110752/Time-Series-Forecasting-from-windowing-to-predicting-in-RapidMiner. Simafore. 5 November 2012. Web. 